\title{
A Multiscale Computational Approach to Dissect Early Events in the Erb Family Receptor Mediated Activation, Differential Signaling, and Relevance to Oncogenic Transformations
}

\author{
Yingting Liu, ${ }^{1, *}$ Jeremy Purvis, ${ }^{4}, *$ Andrew Shih, ${ }^{1}$ Joshua Weinstein, ${ }^{5}$ \\ Neeraj Agrawal, ${ }^{2}$ and Ravi Radhakrishnan $1,2,3,4$ \\ ${ }^{1}$ Department of Bioengineering, University of Pennsylvania, 240 Skirkanich Hall, 210 S. 33rd Street, Philadelphia, PA 19104, \\ USA; ${ }^{2}$ Department of Chemical and Biomolecular Engineering, University of Pennsylvania, Philadelphia, PA, USA; \\ ${ }^{3}$ Department of Biochemistry and Biophysics, University of Pennsylvania, Philadelphia, PA, USA; ${ }^{4}$ Genomics \\ and Computational Biology Program, University of Pennsylvania, Philadelphia, PA, USA; and ${ }^{5}$ Department of Bioengineering, \\ Stanford University, Stanford, CA, USA
}

(Received 22 September 2006; accepted 12 December 2006; published online 2 February 2007)

\begin{abstract}
We describe a hierarchical multiscale computational approach based on molecular dynamics simulations, free energy-based molecular docking simulations, deterministic network-based kinetic modeling, and hybrid discrete/ continuum stochastic dynamics protocols to study the dimermediated receptor activation characteristics of the Erb family receptors, specifically the epidermal growth factor receptor (EGFR). Through these modeling approaches, we are able to extend the prior modeling of EGF-mediated signal transduction by considering specific EGFR tyrosine kinase (EGFRTK) docking interactions mediated by differential binding and phosphorylation of different C-terminal peptide tyrosines on the RTK tail. By modeling signal flows through branching pathways of the EGFRTK resolved on a molecular basis, we are able to transcribe the effects of molecular alterations in the receptor (e.g., mutant forms of the receptor) to differing kinetic behavior and downstream signaling response. Our molecular dynamics simulations show that the drug sensitizing mutation (L834R) of EGFR stabilizes the active conformation to make the system constitutively active. Docking simulations show preferential characteristics (for wildtype vs. mutant receptors) in inhibitor binding as well as preferential enhancement of phosphorylation of particular substrate tyrosines over others. We find that in comparison to the wildtype system, the L834R mutant RTK preferentially binds the inhibitor erlotinib, as well as preferentially phosphorylates the substrate tyrosine Y1068 but not Y1173. We predict that these molecular level changes result in preferential activation of the Akt signaling pathway in comparison to the Erk signaling pathway for cells with normal EGFR expression. For cells with EGFR over expression, the mutant over activates both Erk and Akt pathways, in comparison to wildtype. These results are consistent with qualitative experimental measurements reported in the literature. We discuss these consequences in
\end{abstract}

\footnotetext{
* These authors contributed equally.

Address correspondence to Ravi Radhakrishnan, Department of Bioengineering, University of Pennsylvania, 240 Skirkanich Hall, 210 S. 33rd Street, Philadelphia, PA 19104, USA. Electronic mail: rradhak@seas.upenn.edu
}

light of how the network topology and signaling characteristics of altered (mutant) cell lines are shaped differently in relationship to native cell lines.

Keywords-Epidermal growth factor receptor, Phosphorylation kinetics, Molecular dynamics, Molecular docking, Signal transduction, Stochastic dynamics.

\section{INTRODUCTION}

The Erb family of receptors - the epidermal growth factor receptor (EGFR or ErbB1 or HER1), ErbB2 (or HER2), ErbB3, and ErbB4 - activates a multi-layered network mediating crucial pathways leading to cell proliferation, differentiation, migration and altered metabolism, ${ }^{18,29,53,72}$ in response to activation of the receptor by the epidermal growth factor (EGF), transforming growth factor- $\alpha$ (TGF- $\alpha$ ), and several other related peptide growth factors. ${ }^{18}$

Erb family of receptors are receptor tyrosine kinases (RTKs); RTKs are transmembrane glycoproteins with a cysteine rich ligand-binding extracellular domain, a transmembrane domain important in dimerization, and an intracellular tyrosine kinase domain with a tyrosine rich C-terminal tail. ${ }^{29,53}$ Activation of the ligand-binding domain by an extracellular ligand leads to homodimerization or heterodimerization among the members of the Erb family. The process of ligand-induced dimerization is now quite well understood, based on crystallographic studies. ${ }^{8,25}$ Precisely how this ligand-induced dimerization event is coupled to, and leads to, activation of the intracellular tyrosine kinase domain has also emerged from recent crystallographic studies. ${ }^{75}$ The crystal structure of the EGFRTK domain was first published in 2002, both 
alone and in complex with an inhibitor erlotinib, ${ }^{63}$ and displayed several unique features. While structural studies of the kinase domains from other RTKs (and protein kinases in general) have shown them to be catalytically inactive until an activation event such as ligand-induced dimerization causes them to become autophosphorylated within the activation $\operatorname{loop}^{29}$ and subsequently become locked into a characteristic 'active' conformation, ${ }^{47}$ members of the EGFR family appear to be unique in not requiring activation loop phosphorylation $^{27}$ for their activity. The crystal structure of an unphosphorylated form of the EGFR kinase domain was found to be constitutively locked in the 'active' configuration. ${ }^{63}$ In a recent pioneering crystallographic study, it is suggested that the kinase domain of the EGFR is regulated by a direct allosteric stabilization of the 'active' conformation: ${ }^{75}$ specifically, the kinase-kinase contact through an asymmetric dimer interface allosterically promotes the active conformation. Indeed, such an interface is inferred from (and experimentally realized by) the presence of a high concentration of the kinase domain in the crystals. The study also reported a crystal structure of the V924R mutant of EGFRTK in an inactive form which aligned closely with a previously solved structure of wildtype EGFRTK bound to the inhibitor lapatinib. ${ }^{71}$ The mutation replaces a hydrophobic residue at the dimer interface with a charged group thereby destabilizing the dimer interface. As a consequence, the EGFRTK domain retains its inactive state and relaxes into an alternative crystallographic lattice which does not support the activating asymmetric dimer interface.

It is well established that alteration or over expression of EGFR and ErbB2 - two (of 4) members of the ErbB family of RTKs - is correlated with a variety of clinical cancers. ${ }^{43,58}$ Hence, small molecule RTK inhibitors for EGFRTK and ErbB2 RTK are also of significant interest as cancer therapeutic drugs. Small molecule tyrosine kinase inhibitors (e.g., anilinoquinazoline compounds such as gefitinib and erlotinib), which are ATP analogues, ${ }^{2,3,34,46,51}$ have been shown to exhibit growth inhibitory effects against a wide variety of human tumors. ${ }^{13-17}$ The RTK inhibition approach has shown promise in some clinical trials, but results have been quite mixed. ${ }^{19}$ In particular, despite the promise in a small (demographic) subpopulation with significantly advanced disease, clinical responses to gefitinib and erlotinib varied among population samples. Recently, somatic mutations L834R, L837Q, G685S, del L723-P729 ins S (there is an alternate clinical numbering scheme used in the literature with an offset of 24 residues according to which these mutations are denoted by L858R, L861Q, G719S, del L747-P753 ins S) in the EGFRTK domain were reported to correlate with the clinical response of gefitinib and erlotinib. ${ }^{9,38,42,48}$ In vitro, these EGFR mutants demonstrated enhanced tyrosine kinase activity in comparison to wildtype EGFR and increased sensitivity to inhibition. $3,12,20,21,42,48,49,61$

Although, the underlying biochemical basis involving the drug sensitivity to the mutations is still not clear, the remarkable sensitivity of cell lines carrying gefitinib sensitizing mutants appears not to be centered around inhibitor or ATP binding affinities, but rather on other biochemical mechanisms regulating the EGFRTK activity and EGF-mediated signaling. ${ }^{28}$ In a recent review, the ErbB signaling network is likened to a bow-tie-configured, evolvable network, displaying modularity, redundancy, and control circuitry. ${ }^{18}$ Under this framework, identifying the role and significance of drug sensitizing mutations of ErbB receptors would require a systems level understanding of the signaling network. ${ }^{31-33}$ This would prove to be of enormous value in effectively designing multikinase inhibitors in development for cancer therapy to target many points along the signaling pathway simultaneously. ${ }^{28,35}$ Evidence emerging from biochemical studies points to differential phosphorylation rates associated with different tyrosine phosphorylation sites in the receptor. ${ }^{1,36,61,70}$ Moreover, recent structural studies have indicated that the clinically identified mutations are able to alter the regulatory elements within the receptor such as bypassing the dimer-mediated activation step leading to constitutive activity. Therefore, implications of the effect of such mutations on preferential signaling can be crucial in shaping the signaling response in altered cell lines derived from cancer patients.

In this article, we adopt a multiscale modeling approach (see Flowchart 1 and Table 1) to study the dimer-mediated receptor activation characteristics of EGFRTK. Through these modeling approaches, we are able to extend the prior modeling of EGF-mediated signal transduction by considering specific EGFRTK docking interactions mediated by differential binding

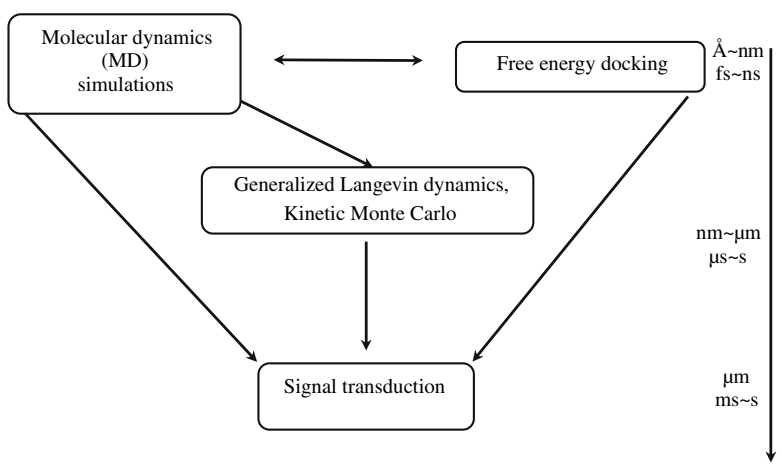

FLOWCHART 1. Scheme for hierarchical multiscale simulations. 


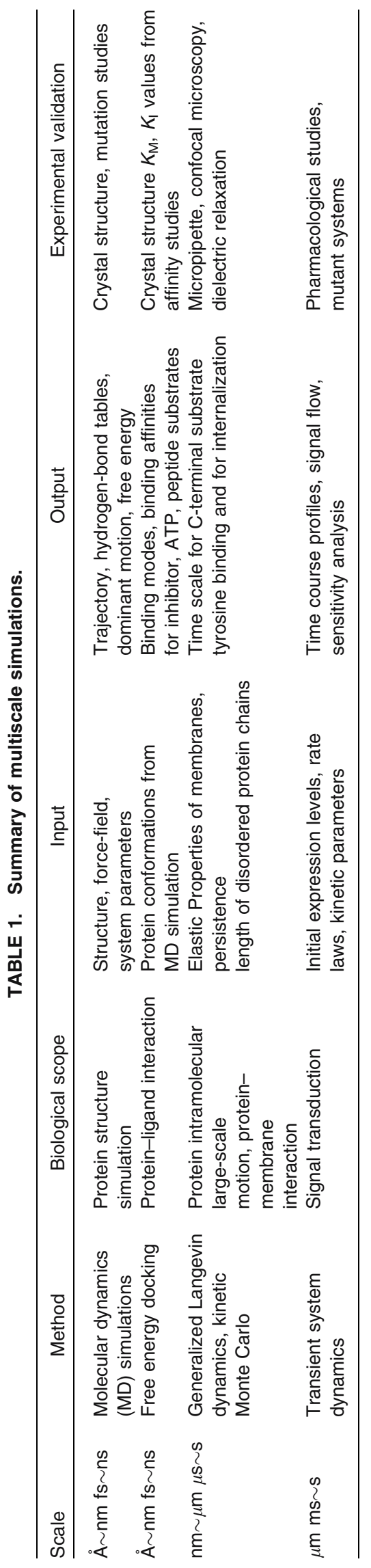

and phosphorylation of different C-terminal peptide tyrosines on the RTK tail. By modeling signal flows through branching pathways of the EGFRTK resolved on a molecular basis, we are able to transcribe the effects of molecular alterations in the receptor (e.g., mutant forms of the receptor) to differing kinetic behavior and downstream signaling response.

\section{OVERALL SUMMARY OF METHODS}

Our multiscale modeling strategy is depicted in the Flowchart 1 and Table 1, (here we provide a brief overview; detailed description is available in the supplementary information S1.1 to S1.6): our hierarchical scheme involves molecular dynamics (see also Section S1.1), free energy docking (see also Section S1.2), hybrid spatial and stochastic modeling ${ }^{69}$ (see also Sections S1.3 and S1.4), and their integration with existing deterministic network models of EGFR-mediated signal transduction (see also Section S1.5). Model approximations and scope are discussed in S1.6.

\section{Molecular Dynamics (MD, see also S1.1)}

Dynamics trajectories of fully atomistic, explicitly solvated systems of wildtype and L834R mutant EGFRTK are obtained and analyzed for specific stabilizing interactions such as hydrogen bonds and saltbridges.

\section{Molecular Docking (see also S1.2)}

Molecular docking is used to predict ligand binding in the absence of a ligand-bound crystal structure and functional affinity data. We employ AutoDock, an automated docking tool designed to predict how small molecules, such as substrates or drug candidates, bind to a receptor of known 3-dimensional structure. ${ }^{45}$ The binding free energy is calculated based on the intermolecular energy between protein and ligands and torsional and solvation free energy of the ligands. ${ }^{45}$ We perform a global conformational search using a multiple conformation docking strategy, in which the protein flexibility is taken into account implicitly.

\section{Signal Transduction (see also S1.3)}

EGF stimulation in a cell results in the simultaneous activation of multiple pathways that are functionally interlinked. ${ }^{36,53,54}$ In the kinetic model we have employed, signaling through the EGFR is modeled by combining three published models and augmented by our own set of reactions and calculations (Section S1.3, 
Figs. S1 and S2). Phosphorylation and docking reactions are modeled according to Kholodenko et al.; ${ }^{37}$ the MAP kinase pathway reactions are modeled after Schoeberl et al. $;^{57}$ Akt and PI3K activation are incorporated into the model as described in Brown et al. ${ }^{7}$ The similar parameterization and topology in these models allowed us to construct a consistent, stable, and comprehensive system with results in good agreement with published experimental data. ${ }^{52}$ Seventeen of these reactions are novel to this work and represent enhanced molecular resolution and detail in EGFR activation, phosphorylation, and docking reactions (Fig. 1 and Table 2). A detailed description of these reactions is provided in Sections S1 and S2. Using the system model, we calculate the state levels of wellknown downstream indicators of cell growth and proliferation, such as phosphorylated ERK and Akt. Each output state is quantified according to peak response, total (integrated) state level, and the time elapsed until peak response.

\section{Intramolecular Diffusional Timescales of C-terminal} Tail Tyrosines in Auto- and Trans-phosphorylation (see also S1.4)

Auto- as well as trans-phosphorylation of specific tyrosine sites in the $\mathrm{C}$-terminal tail of the receptor involves diffusion of the particular tyrosine residue in the C-terminal tail to the active site of the EGFRTK. We model the tail diffusion as a freely joined chain (FJC) consisting of Kuhn segments. ${ }^{22}$ The diffusion coefficient of the tyrosine residue is then calculated using a reptation model ${ }^{22}$ (see S1.4). The reciprocal of the diffusional time of a specified tyrosine residue to reach the active site is taken as the $k_{\text {on }}$ for substrate binding.

\section{Spatial Stochastic Model for Endocytotic Vesicle Nucleation (see also S1.5)}

Clathrin mediated endocytosis is a major mechanism for the down regulation of activated receptor proteins. The assembly of a clathrin coat proceeds via a polymerization of clathrin trimers, ${ }^{55}$ which is observed to coat the endocytotic vesicle. However, it is believed that the clathrin coat alone may not be capable of inducing the required curvature in the membrane. Another protein, epsin, in fact plays a crucial role in inducing the curvature of the membrane. ${ }^{24,26}$ The protein epsin has binding sites in the clathrin lattice and also directly interacts with the membrane. This dual interaction together with the dual interaction of an adaptor protein Ap180 with clathrin and the membrane (Ap180 tethers the clathrin coat to the membrane) is believed to induce the vesicle nucleation and anchor the clathrin lattice to the budding vesicle. Recently, we developed the KMC-TDGL (kinetic Monte Carlo- time-dependent Ginzburg Landau) heterogeneous multiscale approach for studying membrane dynamical processes by combining two different phenomenological theories. ${ }^{69}$ Simulations performed using this approach yields the time evolution of the membrane undulations and deformation in response to the diffusion of membrane bound epsins and a timescale for the nucleation of an endocytotic vesicle. We calculate the timescale for epsin-induced endocytotic vesicle nucleation from our membrane simulations performed using the KMC-TDGL approach. ${ }^{69}$ We combine this timescale for nucleation with the timescales for signal transduction calculated using the network simulations (Section S1.3) to estimate an overall timescale for phosphorylated receptor internalization.

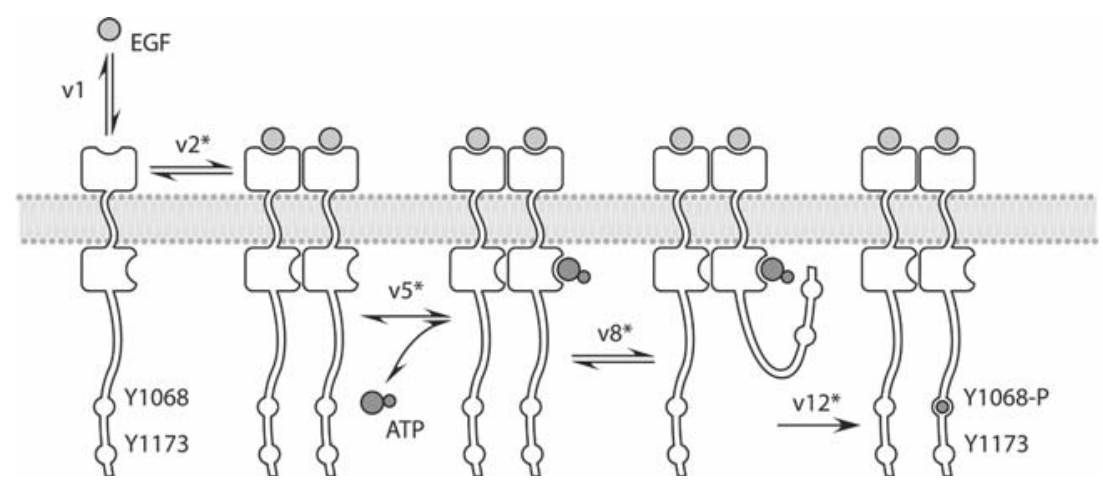

FIGURE 1. Phosphorylation model for EGFR. (v1) EGF binds reversibly to EGFR, which increases the receptor's affinity for other ligand-bound receptors (v2). Basal levels of spontaneous dimerization also occur (v3, v4; not shown). ATP binds reversibly to the catalytic site of the receptor (v5). The transient ATP-bound EGFR must then encounter a free unphosphorylated peptide on either cytoplasmic tail in a diffusion-limited step (v8-v11). (v12) The receptor catalyzes autophosphorylation at either Y1068 or Y1173 (for clarity, only the Y1068 site is shown). Reactions with an asterisk denote more than one reaction involving similar kinetics but differing substrates (see Table 2). 
TABLE 2. Reaction mechanisms and parameterization for EGFR phosphorylation reactions.

\begin{tabular}{|c|c|c|c|c|}
\hline \multirow{2}{*}{$\frac{\text { Reaction }}{\text { v1 }}$} & \multirow{2}{*}{$\frac{\text { Mechanism }}{[E G F R]+[E G F] \rightleftharpoons[E G F R: E G F]}$} & \multicolumn{2}{|c|}{ Parameter values } & \multirow{2}{*}{$\begin{array}{r}\text { Reference } \\
\text { Kholodenko et al. }^{37}\end{array}$} \\
\hline & & $k_{\mathrm{f}}=3 \times 10^{-7} \mathrm{M}^{-1} \mathrm{~s}^{-1}$ & $k_{\mathrm{r}}=3.8 \times 10^{-3} \mathrm{~s}^{-1}$ & \\
\hline v2 & {$[E G F R: E G F]+[E G F R: E G F] \rightleftharpoons\left[E G_{2} R_{2}\right]$} & $k_{\mathrm{f}}=1 \times 10^{-7} \mathrm{M}^{-1} \mathrm{~s}^{-1}$ & $k_{\mathrm{r}}=0.1 \mathrm{~s}^{-1}$ & Kholodenko et al. ${ }^{37}$ \\
\hline$v^{a}$ & {$[E G F R]+[E G F R] \rightleftharpoons\left[E_{G F R}\right]$} & $k_{f}=1 \times 10^{-7} \mathrm{M}^{-1} \mathrm{~s}^{-1}$ & $k_{\mathrm{r}}=1 \times 10^{-3} \mathrm{~s}^{-1}$ & Kholodenko et al. ${ }^{37}$, see $\mathrm{S} 1.3$ \\
\hline$v 5^{b}$ & {$\left[\mathrm{EGFR}_{2}\right]+[\mathrm{ATP}] \rightleftharpoons\left[\mathrm{EGFR}_{2}: \mathrm{ATP}\right]$} & $K_{\mathrm{m}}=2.4 \times 10^{-8} \mathrm{M}^{-1}$ & & ${\text { Brignola et } a .^{5}}^{5}$ \\
\hline$v 8^{c}$ & {$\left[\mathrm{EGFR}_{2}: \mathrm{ATP}\right] \rightleftharpoons\left[\mathrm{EGFR}_{2}: \mathrm{ATP}: \mathrm{Y} 1068\right]$} & $k_{\mathrm{f}}=6.51 \times 10^{5} \mathrm{~s}^{-1}$ & $k_{\mathrm{r}}=769 \mathrm{~s}^{-1}$ & Fan et al. ${ }^{23}$, see $\mathrm{S} 1.3$ \\
\hline $\mathrm{v} 10^{\mathrm{d}}$ & {$\left[\mathrm{EGFR}_{2}: \mathrm{ATP}\right] \rightleftharpoons\left[\mathrm{EGFR}_{2}: \mathrm{ATP}: \mathrm{Y} 1173\right]$} & $k_{\mathrm{f}}=1.98 \times 10^{5} \mathrm{~s}^{-1}$ & $k_{\mathrm{r}}=48.1 \mathrm{~s}^{-1}$ & Fan et $a l .{ }^{23}$, see $\mathrm{S} 1.3$ \\
\hline $\mathrm{v} 12^{\mathrm{e}}$ & {$\left[E G F R_{2}: A T P: Y 1068\right] \rightarrow\left[E G F R_{2}^{Y 1068-P}\right]$} & $k_{\text {cat }}=0.29 \mathrm{~s}^{-1}$ & & Fan et al. ${ }^{23}$ \\
\hline$v 14^{f}$ & 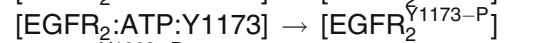 & $k_{\text {cat }}=0.25 \mathrm{~s}^{-1}$ & & Fan et al. ${ }^{23}$ \\
\hline$v 16^{g}$ & {$\left[\mathrm{EGFR}_{2}^{\mathrm{Y1068-P}}\right] \rightarrow\left[\mathrm{EGFR}_{2}\right]$} & $V_{\max }=4.5 \times 10^{-7} \mathrm{M}^{-1} \mathrm{~s}^{-1}$ & $K_{\mathrm{m}}=5.0 \times 10^{-8} \mathrm{M}$ & Kholodenko et al. ${ }^{37}$ \\
\hline
\end{tabular}

${ }^{a}$ An identical rate equation was employed for spontaneous dimerization of EGFR and EGFR:EGF (v4).

${ }^{b}$ Identical rate equations were employed for ATP binding to $\mathrm{EGFR}_{2}{ }^{\mathrm{Y} 1068}$ (v6) and $\mathrm{EGFR}_{2}{ }^{\mathrm{Y} 1173}$ (v7).

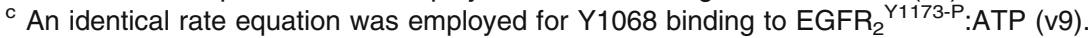

${ }^{d}$ An identical rate equation was employed for $Y 1173$ binding to EGFR 2 Y1068-P:ATP (V11).

e An identical rate equation was employed for phosphorylation of EGFR ${ }_{2}{ }^{\mathrm{Y} 1173-P}: A T P: Y 1068$ (v13).

${ }^{\dagger}$ An identical rate equation was employed for phosphorylation of EGFR ${ }^{Y 1068-P}: A T P: Y 1173$ (v15).

${ }^{g}$ Identical rate equations were employed for dephosphorylation of EGFR ${ }^{\text {Y1173-P }}$ (v17) and EGFR ${ }^{\text {Y1173-P }}$ (v18).

\section{RESULTS}

\section{Dimer Mediated Activation of EGFRTK}

We performed two 10 ns molecular dynamics (MD) simulations of the EGFRTK system in the active and the inactive conformations, respectively. Based on a comparison of the crystal structures - active: erlotinibbound, wildtype ${ }^{63}$ AMP-bound wildtype ${ }^{75}$ inactive: lapatinib-bound wildtype, ${ }^{71}$ V924R mutant $^{75}$-we conclude that the conformational switching from an inactive to an active conformation involves a rotation of the $\alpha$ C-helix of EGFRTK, and the shifting of the activation loop (A-loop) residues to make way for peptide and ATP binding. The recent study of Kuriyan et $a l .{ }^{75}$ has implicated a dimer-mediated activation mechanism for the EGFRTK. That is, loss of interactions upon RTK dimerization leads to switching of the conformational state of the enzyme from inactive to active conformations. On this basis, we hypothesize that specific interactions (H-bonds and salt-bridges) between the residues of the $\alpha \mathrm{C}$-helix and those of the A-loop need to re-organize in the course of the conformational switching from inactive to active states. To elucidate the nature of these interactions, a hydrogen bond analysis focusing on the residues surrounding the A-loop, aC-helix, catalytic loop, and nucleotide-binding loop was carried out using $\mathrm{CHARMM}^{6}$ and $\mathrm{VMD}^{30}$ programs. The MD trajectories were first analyzed in CHARMM with a hydrogen bond cutoff of $2.4 \AA$ and a cutoff angle of $150^{\circ}$. We generated a list of hydrogen bonds that were present in at least $60 \%$ of the trajectory. These hydrogen bonds were then visualized in VMD and any non-significant bonds were removed to reveal the stabilizing $\mathrm{H}$-bonds. During the $\mathrm{H}$-bond analysis, acidic and basic residues formed strong hydrogen bonds, similar to salt-bridges. The A-loop and $\alpha \mathrm{C}$-helix conformations were stabilized by internal hydrogen bonds (i.e., both the residues participating in the hydrogen bond are within the loop/ helix), as well as external hydrogen bonds (i.e., one residue is in the loop/helix, the other is not). The residues participating in these stabilizing interactions are predominantly acidic and basic residues. A comparison of the specific interactions showed that the number of external interactions stabilizing the A-loop and $\alpha \mathrm{C}$ helix in the active conformations far out-numbered those in the inactive conformations (see Fig. 2 and Table 3). Intriguingly, the small number of stabilizing interactions holding the kinase in the inactive are predominantly located proximal to the dimer-interface residues (Table 3), which lends further credibility to the allosteric activation mechanism ${ }^{75}$ of the wildtype. Namely, that loss dimer-interface residues will alter the surrounding stabilizing bond network, which likely leads to the destabilization of the inactive conformation and triggers the conformational shift to the active conformation.

\section{Constitutive Activation of the L834R Mutant}

The clinically relevant mutations del724-729, and L834R are structurally in distinct parts of the enzyme but overall both lead to enhanced activity. ${ }^{10,42,48,61}$ Our delineated pattern of stabilizing interactions in Table 3 serves as a platform for unifying the effects of these mutations at a structural level. The small number of stabilizing interactions holding the kinase in the inactive conformation also overlaps with the residues associated with several clinically relevant mutations. The deletion mutant interferes directly 

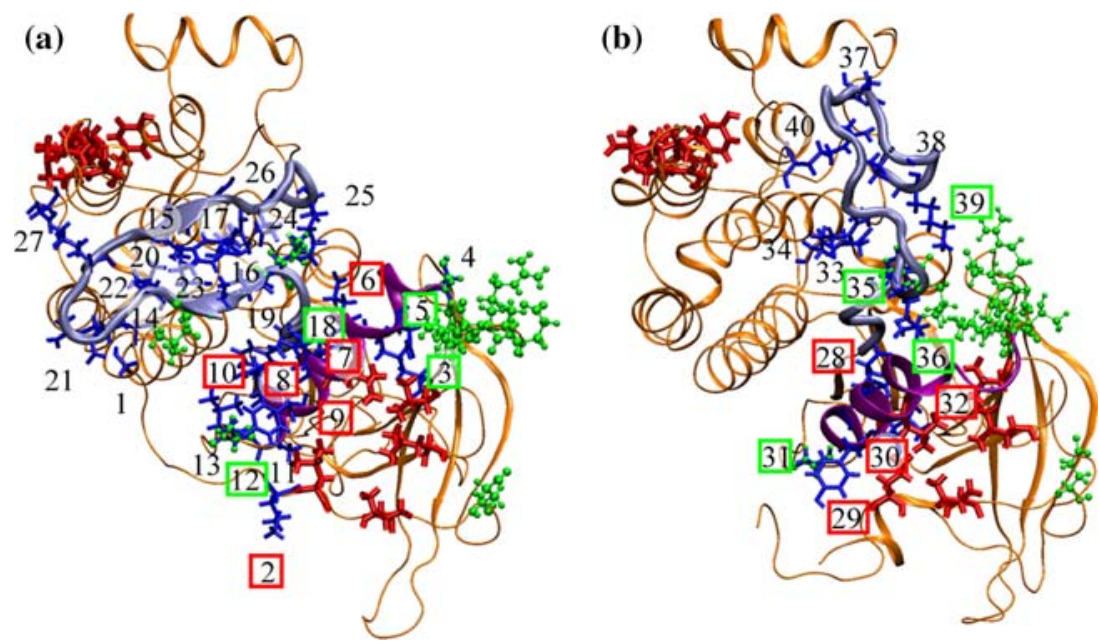

FIGURE 2. Visualization of the stabilizing residues external to A-loop and $\alpha \mathrm{C}$-helix (shown in blue), dimer interface residues (shown in red) and clinical mutations (shown in green) of both the active (panel a) and inactive (panel b) EGFR tyrosine kinases. The A-loop is highlighted in sky blue while the $\alpha \mathrm{C}$-helix is shown in purple, the remainder of the protein is colored orange. The external stabilizing residues for the EGFR tyrosine kinases are numbered from 1-40, with 1-27 corresponding to residues in the active state while $\mathbf{2 8 - 4 0}$ correspond to the stabilizing residues in the inactive state. The stabilizing residues that are potentially affected by dimerization are highlighted in red boxes, and the residues that are potentially affected by the clinical mutations are highlighted in green boxes (See also Table 3). The inactive conformation has fewer stabilizing bonds holding the conformation stable: 13 stabilizing residues as opposed to 27 stabilizing residues. Therefore, the loss of interactions triggered by either mutation or dimerization will have a greater effect upon the stability of the inactive kinase and allow a conformation change. The numbered labels correspond to the residue numbers as follows: 1:672 2:679 3:721 4:728 5:732 6:734 7:737 8:738 9:740 10:741 11:743 12:744 13:745 14:808 15:810 16:812 17:832 18:834 19:836 20:838 21:840 22:843 23:845 24:848 25:851 26:867 27:932 28:738 29:740 30:742 $31: 74432: 753$ 33:812 34:813 35:834 36:836 37:846 38:848 39:851 40:865.

with the dimer interface and the $\mathrm{R}$ substitution of $\mathrm{L}$ at 834 as well as $\mathrm{R}$ substitution of $\mathrm{Q}$ in 837 destabilizes the specific (external $\mathrm{H}$ bonds) interactions associated with A-loop and $\alpha \mathrm{C}$-helix in the inactive but not the active conformations. Thus, these mutations have a deleterious effect (see Table 3 ) in stabilizing A-loop and $\alpha \mathrm{C}$-helix conformations in the inactive state, hence stabilizing the active conformation. Indeed, in one of our MD trajectories initiated from the active conformation of the wildtype monomer RTK, we observed the motion of the enzyme towards the inactive state in $10 \mathrm{~ns}$. In this case, a key interaction between two residues in the $\alpha \mathrm{C}$-helix and the A-loop (salt-bridge between GLU851 and LYS734, see also Table 3) was broken. In stark contrast, a 12 ns MD trajectory of the L834R mutant system (under similar conditions) stayed stable in the active conformation. The collective evidence from these observations points to a constitutively active L834R mutant RTK system (i.e., activated in monomer and dimer states in the presence or in the absence of an EGF ligand) and a dimer-mediated activation of wildtype RTK (i.e., activated only in the dimer state in the presence of the EGF ligand).

Experimental observation of constitutive activation in the gefitinib-sensitive EGFR mutants has been recorded independently in several studies, which report significantly elevated basal phosphorylation (in the absence of the stimulating ligand) of the mutant systems in comparison to the wildtype. ${ }^{10,11,48,61}$

\section{Ligand and Substrate Binding Affinities for EGFRTK}

We determine the ATP and erlotinib binding modes and binding affinities based on our multiple conformations docking strategy (Section S1.2) and analyze several low-energy clusters of ligand conformations. For both wildtype and L834R mutant EGFRTK systems, one lead erlotinib binding conformation is found to be closely aligned with that in the crystal structure: the hydrogen bonds between the N1 of the quinazoline and Met769 are present and so is the interaction between Thr766 and the quinazoline nitrogen atom (N3). The binding modes for ATP and erlotinib are also consistent with the existing pharmacophore model for ATP binding to kinases. ${ }^{66,67,74}$ Significantly, a second binding mode, which has a higher affinity to the mutant kinase L834R, is revealed in our calculations. This mode still blocks the ATP binding pocket and in addition shows an additional interaction between erloitnib and the $\mathrm{R} 834$ residue. The effect is a higher calculated binding affinity for erlotinib binding to L834R $(-9.4 \pm 0.4 \mathrm{kcal} / \mathrm{mol})$ in comparison to wildtype $(-7.3 \pm 0.5 \mathrm{kcal} / \mathrm{mol})$.

In order to explore the C-terminal peptide-substrate-bound pre-catalytic conformation, we also dock 
Table 3. External stabilizing interactions in inactive and active conformations, dimer-interface residues, and clinical mutations.

\begin{tabular}{|c|c|c|c|}
\hline \multicolumn{2}{|c|}{ alphaC-helix } & \multicolumn{2}{|c|}{ A-loop } \\
\hline \multicolumn{4}{|c|}{ Stabilizing Salt Bridges } \\
\hline Active & Inactive & Active & Inactive \\
\hline 2 & 1 & 3 & 2 \\
\hline \multicolumn{4}{|c|}{ Stabilizing H-bonds } \\
\hline 5 & 2 & 7 & 3 \\
\hline \multicolumn{4}{|c|}{ alphaC-helix (Residues 729 to 743) } \\
\hline \multicolumn{2}{|c|}{ Active } & \multicolumn{2}{|c|}{ Inactive } \\
\hline \multicolumn{4}{|c|}{ Stabilizing Salt Bridges } \\
\hline GLU 734 & LYS 851 & GLU 738 & LYS 836 \\
\hline GLU 738 & LYS 721 & & \\
\hline \multicolumn{4}{|c|}{ Stabilizing H-bonds } \\
\hline ASN $732 \mathrm{HN}$ & SER $728 \mathrm{O}$ & TYR $740 \mathrm{O}$ & $\begin{array}{l}\text { SER } 744 \\
\text { HN,HG1 }\end{array}$ \\
\hline $\begin{array}{c}\text { GLU } 738 \\
\text { OE1,2 }\end{array}$ & PHE $832 \mathrm{HN}$ & MET $742 \mathrm{O}$ & LEU $753 \mathrm{HN}$ \\
\hline TYR $740 \mathrm{O}$ & SER 744 HG1 & & \\
\hline VAL $741 \mathrm{O}$ & VAL $745 \mathrm{HN}$ & & \\
\hline ALA $743 \mathrm{O}$ & LEU $679 \mathrm{HN}$ & & \\
\hline \multicolumn{4}{|c|}{ Activation Loop (Residues 831 to 852) } \\
\hline \multicolumn{2}{|c|}{ Active } & \multicolumn{2}{|c|}{ Inactive } \\
\hline \multicolumn{4}{|c|}{ Stabilizing Salt Bridges } \\
\hline LYS 843 & ASP 932 & LYS 836 & GLU 738 \\
\hline GLU 848 & ARG 812 & GLU 848 & ARG 865 \\
\hline LYS 851 & GLU 734 & & \\
\hline \multicolumn{4}{|c|}{ Stabilizing H-bonds } \\
\hline PHE $832 \mathrm{HN}$ & $\begin{array}{c}\text { GLU } 738 \\
\text { OE } 1,2\end{array}$ & $\begin{array}{c}\text { LEU } 834 \\
\text { HN }\end{array}$ & ASP 813 OD1 \\
\hline LEU $834 \mathrm{O}$ & $\begin{array}{c}\text { ARG } 812 \\
\text { HH12 }\end{array}$ & $\begin{array}{l}\text { HIS } 846 \\
\text { NE2 }\end{array}$ & ARG 865 HH11 \\
\hline LYS 836 HZ2 & ASP 737 OD2 & LYS $851 \mathrm{O}$ & $\begin{array}{l}\text { ARG } 812 \\
\text { HH11,21 }\end{array}$ \\
\hline $\begin{array}{l}\text { LYS } 836 \\
\text { O,HN }\end{array}$ & $\begin{array}{c}\text { VAL } 810 \\
\text { HN,O }\end{array}$ & & \\
\hline LEU $838 \mathrm{HN}$ & ARG $808 \mathrm{O}$ & & \\
\hline ALA $840 \mathrm{HN}$ & GLY $672 \mathrm{O}$ & & \\
\hline $\begin{array}{c}\text { TYR } 845 \\
\text { O,HN }\end{array}$ & $\begin{array}{c}\text { TYR } 867 \\
\text { HN,O }\end{array}$ & & \\
\hline \multicolumn{4}{|c|}{$\begin{array}{l}\text { Dimer Interface Residues } \\
\text { L680 I682 L736 L758 V762 I917 Y920 M921 V924 M928 } \\
\text { Clinical Mutations } \\
\quad \text { L834R del723-728 S744I L837Q E685G G695S }\end{array}$} \\
\hline
\end{tabular}

EGFRTK substrate peptides to the catalytic site. First, we focus on an optimal peptide sequence EEEYFEL as a control. This optimal sequence is selected for a maximal value in $k_{\mathrm{cat}} / K_{\mathrm{M}}$ among EGFRTK substrate peptides. ${ }^{60}$ Based on $500-1000$ different genetic algorithm runs for docking of this peptide to EGFRTK, we select the best binding conformation for binding as one that yields the lowest binding free energy simultaneously with the correct orientation for the tyrosine binding into the active site. The latter criterion is essential in ensuring that $k_{\mathrm{cat}}$ is not significantly compromised in obtaining a stronger binding. We depict in Fig. 3, the peptide conformation bound to the active site, which is seen to be ideally poised for a two-metalion catalyzed phosphoryl transfer from ATP to the bound tyrosine: ${ }^{44,64}$ i.e., the relative positions of the ATP, $\mathrm{Mg}^{2+}$ ions, and the catalytic aspartates are in good alignment. Encouraged by this result, we performed peptide docking simulations for two substrate peptides (each being seven amino acids long) corresponding to the Y1068 (VPEYINQ) and Y1173 (NAEYLRV) sites of the C-terminal tail for both wildtype and L834R mutant structures. Based on a similar criteria of lower binding free energy and optimal catalytic geometry, we calculated the peptide binding affinity corresponding to sites Y1068 and 1173 for wildtype and L834R mutant systems. Upon mutation, the calculated $K_{\mathrm{M}}$ for the Y1068 peptide decreased 20-fold (calculated $\Delta \Delta G$ [wildtype $\rightarrow$ L834R] $=-2.2 \mathrm{kcal} / \mathrm{mol}$ ), while the calculated $K_{\mathrm{M}}$ for the Y1173 peptide increased 4-fold (calculated $\Delta \Delta G$ [wildtype $\rightarrow \mathrm{L} 834 \mathrm{R}$ ] $=+0.9 \mathrm{kcal} / \mathrm{mol}$ ). Analysis of the binding modes reveals that the relative alignment of the tyrosine with respect to the catalytic site is ideal (2.2 $\AA$ in the observed distance $\xi$ between Tyr $\mathrm{OH}$ oxygen and Asp $813 \mathrm{O}_{\delta 2}$ ) for the two peptide tyrosines binding to wildtype, with the two mutant systems showing a $0-1.5 \AA$ deviation. The differences can translate into differences in $k_{\text {cat }}$ values associated with the tyrosine phosphorylation. Assuming a harmonic energy profile along the reaction coordinate $\xi$ with a force constant $K_{\xi}$, the work done in reducing this distance is given by $1 / 2 K_{\xi} \xi^{2}$. The value of $K_{\xi}$ is obtained by recording the fluctuations of $\xi$ in the MD simulations $\left(K_{\xi}=k_{\mathrm{B}} T / \sigma_{\xi}^{2}=0.77 \mathrm{kcal} / \mathrm{mol} / \AA^{2}\right.$ for the wildtype and $1.01 \mathrm{kcal} / \mathrm{mol} / \AA^{2}$ for L834R), where $\sigma_{\xi}$ is the standard deviation associated with the fluctuations in $\xi$ in the trajectories). The normalized values of $k_{\text {cat }} / K_{\mathrm{M}}$ can then be compared for the four systems

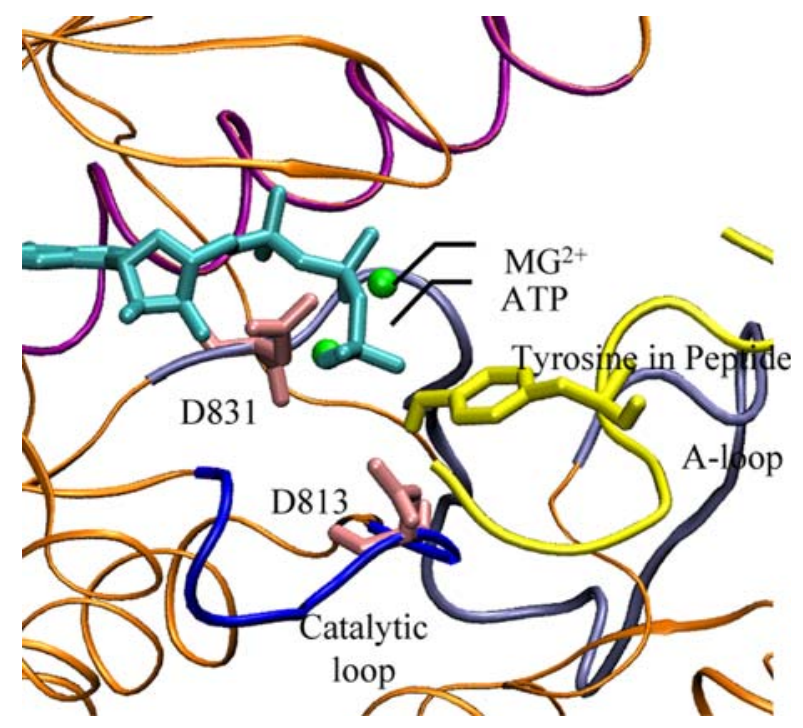

FIGURE 3. Binding modes for ATP (cyan) and the optimal peptide sequence (yellow) in the EGFRTK domain. The geometry of the active site is consistent with that prescribed for an ideal two-metal-ion catalyzed mechanism and reveals a state that is poised for the phosphoryl transfer reaction. The alignment of the two divalent $\mathrm{Mg}^{2+}$ ions as well as the catalytic aspartates D813 and D831 are also shown. 
at the same value of $\xi=2.2 \AA$ ); the relative increase in $k_{\text {cat }} / K_{\mathrm{M}}: 1068$ for the $\mathrm{L} 834 \mathrm{R}$ in comparison to the wildtype is 20 -fold (increase by 1 -order of magnitude), while the relative decrease for the 1073 is 200 -fold (decrease by 2 -orders of magnitude). This differential sensitivity translates into differences in phosphorylation of the associated tyrosine sites, which ultimately leads to differential signaling and down stream activation in the EGFR network: the Y1173-phosphorylation is associated Shc mediated ERK activation and Y1068-phosphorylation is associated with Akt activation. ${ }^{36,61}$

\section{Time-scales Associated with Diffusion of Substrate Tyrosines Binding Prior to Auto- and Trans- phosphorylation and with Endocytotic Vesicle Nucleation}

\section{C-terminal Tail Diffusion}

Using the method outlined in Section S1.4, we calculate the diffusional time scale for each of the seven tyrosine residues present on the $\mathrm{C}$-terminal tail of EGFRTK to reach the active site (Table 4). The timescales for diffusion are in $\mu$ s and not very different for the different tyrosine sites to bind to the active site prior to auto- (head) or trans- (tail) phosphorylation. For each case, the reciprocal of the corresponding timescale is taken as the on-rate $\left(k_{\text {on }}\right)$ of the tyrosine binding to the active site. The off-rate is calculated as $k_{\text {off }}=k_{\text {on }} \times \exp \left(+\Delta G / k_{\mathrm{B}} T\right)$, where $\Delta G$ is the binding free energy of the substrate to the active site; here it is assumed that the binding process is diffusion-limited with no significant additional barriers. The values for $\Delta G$ for each C-terminal tyrosine is obtained from the analogous data on peptide substrate affinities $K_{\mathrm{M}}$ published in the literature: ${ }^{5,23} \Delta G / k_{\mathrm{B}} T=-\ln \left(K_{\mathrm{M}}\right)$. These calculated values are used in the model for signal transduction described in Section 2.3 and provided in Table 2 .

TABLE 4. Diffusional time for C-terminal tyrosines to reach the active site of EGFRTK

\begin{tabular}{lcc}
\hline $\begin{array}{l}\text { Tyrosine } \\
\text { residue }\end{array}$ & \multicolumn{2}{c}{ Diffusional time to the active site $(\mu \mathrm{s})$} \\
\cline { 2 - 3 } & $\begin{array}{c}\text { Head EGFRTK } \\
\text { (auto-phosphorylation) }\end{array}$ & $\begin{array}{c}\text { Tail EGFRTK } \\
\text { (trans-phosphorylation) }\end{array}$ \\
\hline Y992 & 0.34 & 0.65 \\
Y1045 & 1.72 & 2.54 \\
Y1068 & 2.64 & 3.68 \\
Y1086 & 3.49 & 4.7 \\
Y1101 & 4.26 & 5.61 \\
Y1148 & 7.26 & 9.08 \\
Y1173 & 9.16 & 11.22 \\
\hline
\end{tabular}

\section{Endocytotic Vesicle Nucleation}

In our earlier work, we identified different regimes conducive to nucleation of an endocytotic vesicle by exploring a range of parameters associated with the membrane adsorbed proteins, such as surface density of the protein on the membrane, and the intrinsic curvature of the membrane induced by the proteinmembrane interaction. ${ }^{69}$ Here, we report our results specifically for epsins interacting with the membrane. Consistent with the experimental values of Ford et al., we assume that the intrinsic membrane curvature induced by the epsin molecules falls in the range of $10 \mu \mathrm{m}^{-1}$. We further assume that the distance from a given epsin molecule that this curvature effect decays is four times the epsin diameter. A depiction of our findings for an epsin surface density (on the membrane) of 60 per $\mu \mathrm{m}^{2}$ is given in Fig. 4, in which in the time-course of $250-400 \mathrm{~s}$, the nucleation of a vesicle is observed (see snapshots in panels B-D). Panels E and $\mathrm{F}$ provide the corresponding spatial and orientational correlations. ${ }^{69}$ The necessary condition for nucleation is the persistence of orientational (hexagonal) ordering of the membrane-adsorbed epsin molecules (see caption). ${ }^{69}$ Our results have suggested an interesting mechanism for the epsin-mediated membrane invagination. Namely, the invagination occurs through a nucleation event, which involves the co-localization and hexagonal ordering of several epsin molecules on the membrane. Based on our results (Fig. 4), the timescale for nucleation of such an event is $250-400 \mathrm{~s}$.

As described in Section 2.3, receptor activation, which is eventually followed by receptor internalization (via endocytosis), results in triggering many signal transduction cascades, one of which being the PLC $\gamma$ pathway. ${ }^{4,72}$ The PLC $\gamma$ pathway is accompanied by a transient release of calcium ions from intracellular stores. Models for the transient release of calcium have been developed on the basis of several published experimental studies. ${ }^{39,59}$ We work under the hypothesis that the signal transduction in the endocytosis pathway is coupled to membrane deformation via two functional interactions: (1) the direct induction of curvature via the interaction of the membrane lipids with epsin, ${ }^{24,50,56,62}$ and (2) through the assembly of the clathrin coat, ${ }^{56,73}$ which is triggered by an increase in the local concentration of $\mathrm{Ca}^{2+}$ ions released in the PLC $\gamma$ pathway. ${ }^{4}$ In the latter case, above a threshold value of $\mathrm{Ca}^{2+}$ concentration the clathrin monomers spontaneously self-assemble to form a lattice. ${ }^{56,73}$ This leads to the following mechanism for endocytosis. The transient increase in calcium concentration at the site of the activated receptor helps promote clathrin polymerization and coat assembly. The adapter protein Ap180 tethers the clathrin lattice on the membrane. 

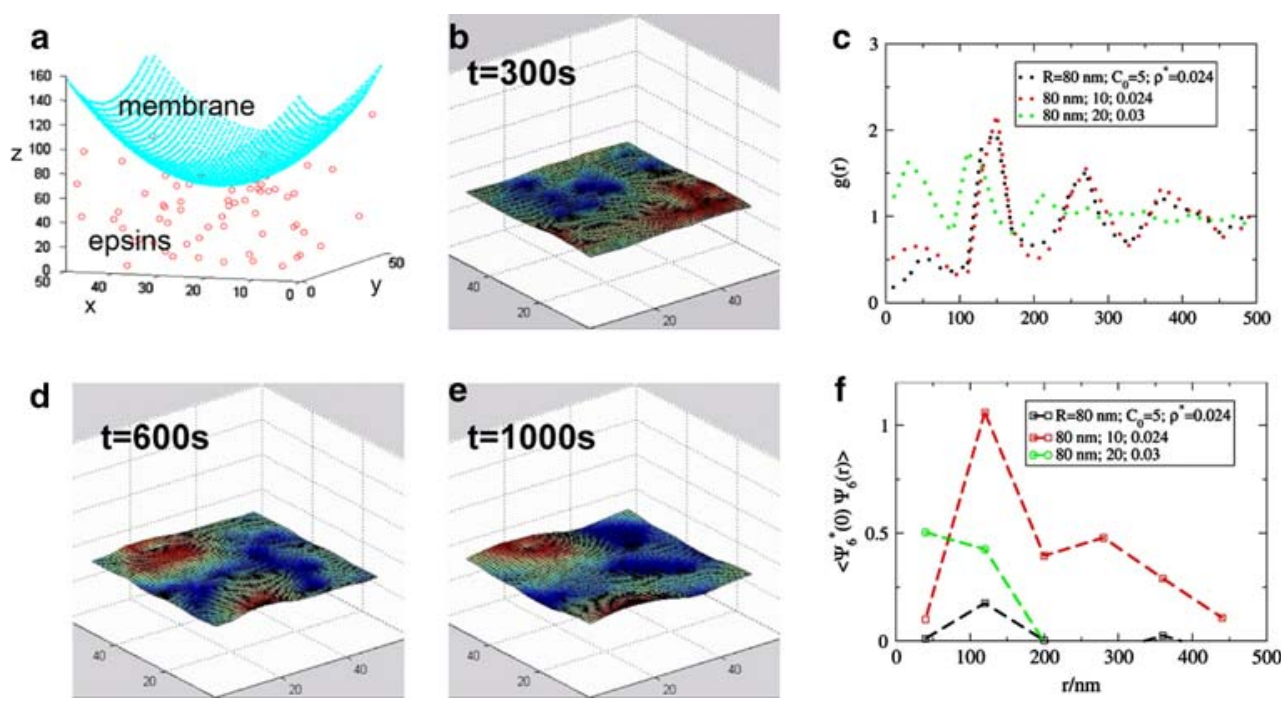

FIGURE 4. (a) Depiction of the system of epsin molecules interacting with the membrane. (b-d) Snapshots of the system at different time points (the epsin positions are not shown for clarity). The blue regions are recessed and red regions are elevated in the membrane. The nucleation of a budding vesicle in the membrane is evident in panel. (d, e) Spatial correlation function for the membrane adsorbed epsins. (f) Orientational correlation function for the membrane adsorbed epsins. The correlation functions are provided for 3 simulation runs at different epsin densities $\left(\rho^{\star}\right.$, dimensionless) and values of the induced curvature ( $c_{0}$ expressed in $\left.\mu \mathrm{m}^{-1}\right)$. The value of a dimensionless density $\left(\rho^{\star}=0.024\right.$ corresponds to 60 epsins per $\mu \mathrm{m}^{2}$. The snapshots correspond to the red symbols in panels e and $\mathrm{f}\left(\rho^{*}=\mathbf{0 . 0 2 4}\right.$ and $\left.c_{0}=10 \mu \mathrm{m}^{-1}\right)$. For this case, there is persistence of orientational correlations (hexagonal ordering), see panel $f$. For the other two cases in panels e and $f$ (for which snapshots are not shown), no nucleation and no significant orientational ordering is observed.

The vertices of the hexagonal lattice of the clathrin coat provide binding sites for epsins. Thus, in the vicinity of the activated receptor, the membrane adsorbed epsins assume a spatial conformation with persistent orientational correlations induced by the clathrin lattice. This provides the condition for a nucleation event leading to formation of the endocytotic vesicle. (We note that according to this mechanism, unactivated receptors can also internalize; however, in the absence of the transient calcium surge, which only occurs upon receptor activation, the clathrin coat assembly may represent a slower step).

An estimate for the timescale of receptor internalization $\tau$ (internalization) is therefore obtained by the sum of timescales for receptor phosphorylation at Y1045, PLC $\gamma$ activation, calcium release, clathrin coat assembly, and nucleation of epsin-mediated vesicle formation.

\section{Differential Signaling through EGFRTK}

By employing the deterministic model described in Section 2.3 (see Figs. 1 and S1, Table 2) we have calculated transient evolution of the EGF-mediated signaling network under a variety of initial conditions and parameter values. In particular, we have focused on two pools of normal ([EGFR] $=100 \mathrm{nM}$ or 33,000 receptors per cell) and over expressed ([EGFR] $=$ $2000 \mathrm{nM}$ or 660,000 receptors per cell) EGFR con- centrations, each in the absence (serum starved, $[E G F]=0 \mathrm{nM})$ and presence (serum cultured, $[\mathrm{EGF}]=8 \mathrm{nM})$ of the EGF ligand. A typical timecourse resulting from our network simulations is provided in Fig. S3, in which the kinetics of phosphorylation of EGFRTK and down-stream markers Ras, Raf, PLC $\gamma$, PI3K, and Akt are depicted. Since our molecularly resolved model (Fig. 1) is currently focused on differential signaling through Y1068 and Y1173 phosphorylation sites of EGFRTK, we track the integrated response of ERK and Akt phosphorylation levels in our simulations. Each output state was quantified according to the peak level of phosphorylation over the simulated time of $1000 \mathrm{~s}$. Specifically, we perform a two-dimensional scan over $k_{\text {cat }} / K_{\mathrm{M}}$ values associated with Y1068 and Y1173 phosphorylation in which the respective $k_{\text {cat }} / K_{\mathrm{M}}$ values are allowed to deviate from their default (wildtype) value over a logarithmic range of five log units. The result is a twodimensional matrix in which each element represents output from a single simulation involving a unique pair of parameters (see Fig. 5).

The simulated response of the EGFR network also enables us to estimate a timescale for receptor internalization $\tau$ (internalization). The typical timescale for EGFRTK phosphorylation is in the range of $10 \mathrm{~s}$, while that for PLC $\gamma$ activation is $50 \mathrm{~s}$ (see Figs. S3 and $\mathrm{S} 4)$. The calcium release from intracellular stores through the activation of IP3R (IP3 receptors) is re- 
EGFR Normal Expression

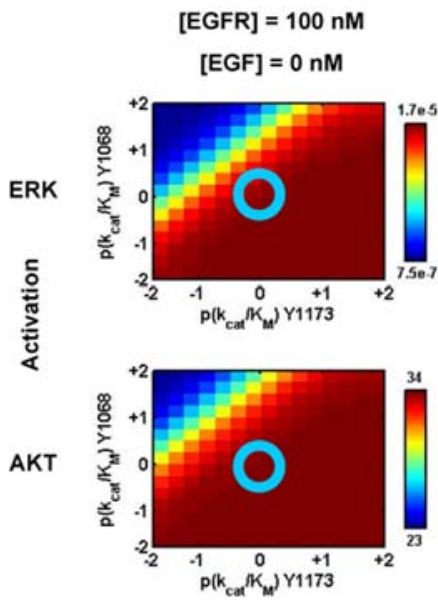

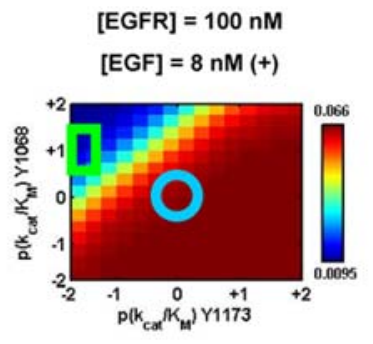

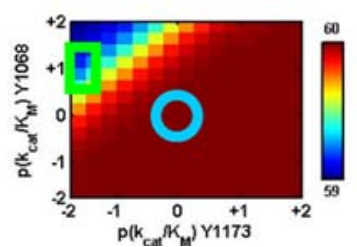

EGFR Over Expression

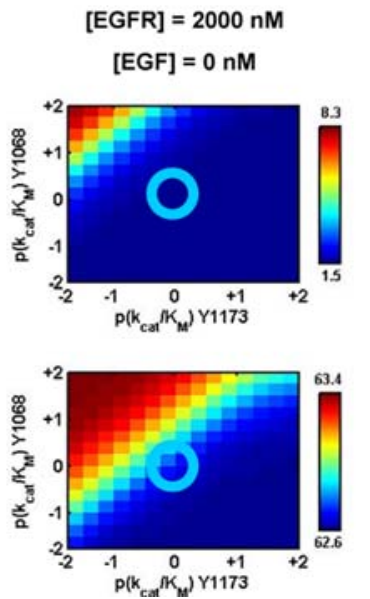

[EGFR] $=2000 \mathrm{nM}$

[EGFR] $=2000 \mathrm{nM}$

$[E G F]=8 \mathrm{nM}(+)$
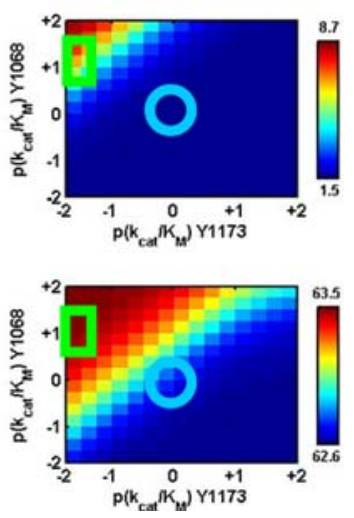

FIGURE 5. Calculated ERK and Akt phosphorylation levels in units of nM (peak-levels over the time course of $1000 \mathrm{~s}$ ) under serum starved (EGF-) and serum cultured (EGF+) conditions for cell types with normal EGFR expression and EGFR over expression. In each panel the Y1173 rate of phosphorylation $\left(k_{\mathrm{cat}} / K_{\mathrm{M}}\right)$ is varied along the $x$-axis (value relative to wildtype EGFRTK) and the Y1068 rate of phosphorylation $\left(k_{\mathrm{cat}} / K_{\mathrm{M}}\right)$ is varied along the $y$-axis. In each panel, the blue circle denotes the wildtype levels and the green square denotes those relevant to the L834R mutant cell lines.

ported to occur within a short timescale of $10 \mathrm{~s} .{ }^{59}$ While the timescale for clathrin coat self-assembly under high calcium concentration is not known, it is assumed here that this is a rapid process. According to our results from the spatial stochastic simulations (Section 3.4), the epsin mediated endocytotic vesicle nucleation in the presence of the clathrin coat is calculated to occur in a timescale $250-400 \mathrm{~s}$. Further, assuming that the stabilization of the budding endocytotic vesicle and the vesicle pinch-off process catalyzed by dynamin are not rate-limiting, the estimated time for vesicle internalization upon EGF ligand binding is $\approx 450 \mathrm{~s}(\approx 8 \mathrm{~min})$. We note that these timescales from our simulations are ball park estimates and we have not yet attempted a careful comparison with timescales from experimental measurements.

\section{DISCUSSION AND CONCLUSIONS}

Based on an integration of a large body of knowledge accumulated over the years, the modular nature of EGFR-mediated signaling has become apparent. ${ }^{18,31}$ Through a hierarchical multiscale modeling scheme, we have realized a molecular resolution to this system-level view, which can potentially augment our predictive as well as comprehensive capabilities and rationalize the changes in early signaling characteristics of altered and mutant cell lines. Our overall findings are summarized below.

Analysis of stabilizing interactions in our molecular dynamics simulations have corroborated a dimer- mediated activation mechanism for the EGFRTK. That is, loss of key stabilizing interactions in the inactive conformation of the kinase upon dimerization results in destabilizing the inactive state and triggering a conformational change to the active state. Moreover, our results point to a constitutively active L834R mutant. That is, even in a monomer state, the RTK of the L834R mutant system is stabilized in an active conformation and can trigger signaling through the EGFR network by auto-phosphorylation of its C-terminal tail tyrosine residues in an EGF (ligand) independent manner. These results are consistent with the existing biochemical data which have $\mathrm{e}^{10,42,48,61,75}$ qualitatively shown that the phosphorylation of Cterminal tail tyrosines of the L834R mutant in the absence of EGF stimulation is comparable to wildtype values in the presence of EGF stimulation at EGF concentrations of $30-100 \mathrm{ng} / \mathrm{ml}$.

Our results from the free energy docking simulations suggest enhanced binding of the inhibitor erlotinib to the L834R mutant system in comparison to wildtype binding. Our results also reveal preferential binding and phosphorylation of certain C-terminal tyrosine substrates (but not others) to the L834R mutant in comparison to the wildtype: that is, the calculated change in the turn over rate $\left(k_{\mathrm{cat}} / K_{\mathrm{M}}\right)$ upon the L834R mutation is markedly different for the Y1173 peptide in comparison to the Y1068 peptide. This results in a differential sensitivity at the receptor level. Specifically, the estimated increase in $k_{\text {cat }} / K_{\mathrm{M}}$ for the Y1068 peptide is $\sim 10$-fold in comparison to a decrease of $\sim 100$-fold for the Y1173 peptide. Thus, 
we expect to see a net increase in phosphorylation of Y1068 and a net decrease in phosphorylation of Y1173 for the L834R mutant, in comparison to wildtype levels. The biochemical results of Sordella et al. ${ }^{61}$ and Chen et al. ${ }^{10}$ corroborate this view qualitatively; in these studies, the immunoblots measuring the degree of phosphorylated receptor at the Y1173 site show a modest decrease for the L834R mutant in contrast to a strong increase for the Y1068 site, both in comparison to their respective wildtype measurements.

Our systems level simulation illustrates how the altered affinities at the receptor level for the wildtype and L834R translates into changes in the downstream response. In Fig. 5, we explored how the variation in Y1173 and Y1068 phosphorylation affects the peak responses of ERK and Akt for two different EGFR concentrations, each in the absence or presence of EGF stimulation. The calculated responses for the wildtype values are highlighted by the blue circles at the origin of each of the panels. The responses for L834R are identified by the green square centered at a $\left(k_{\text {cat }} / K_{\mathrm{M}}\right) /\left(k_{\text {cat }} / K_{\mathrm{M}}\right)_{\text {wildtype }}$ value of $1 / 100\left(-2\right.$ in $\log _{10}$ units) for Y1073 and 10 (+1 in $\log _{10}$ units) for Y1068. Owing to the constitutively active characteristic of the L834R mutant, the signals relevant for the L834R system (even in the absence of EGF) are analogous to those in the panels with EGF stimulation. As indicated by the color maps in these two scans, increasing the degree of phosphorylation of the Y1173 site (positive $x$-direction) leads to an overall increase in ERK response. This finding is consistent with our model which is based on Y1173 being an important docking site for the She and Grb-2 adaptors that lead to Ras activation and subsequent Erk phosphorylation. For normal EGFR expression, the calculated ERK response in the absence of EGF is low for the L834R mutant $(0.01 \mathrm{nM})$ but negligible (zero) for the wildtype. In the presence of EGF the ERK response for L834R is 7-fold lower than that for the wildtype. For over expressed EGFR, the ERK activation is 7-fold higher in L834R compared to wildtype both in the presence as well as in the absence of EGF stimulation. Thus, ERk stimulation is higher in EGFR overexpressed cells. The Akt response relative to the wildtype is 2-fold higher for the mutant cells under no EGF stimulation and about the same under EGF stimulation for normal EGFR expression. The Akt response does not seem to be affected by mutation for high EGFR expression either in the presence or absence of EGF.

Our calculated responses for ERK and Akt signaling for normal EGFR expression agree with the qualitative experimental observations of Sordella et al. ${ }^{61}$ and Tracy et al.: ${ }^{65}$ namely, that the ERk stimulation levels decrease and the Akt levels are maintained for the mutant cells with normal EGFR expression levels. This preferential activation of Akt in L834R mutant cell lines observed in these experiments is consistent with the systems level predictions from our simulations. Moreover, our calculations predict that the preferential Akt activation is not featured in EGFR over-expressed cells.

In light of the overall agreement between simulations and experiment, we can begin to explore the origins of inhibitor sensitivity in L834R mutant cell lines. The enhanced sensitivity of erlotinib to L834R mutant arises from a combination of several effects: (1) the mutant system has a higher binding affinity to the inhibitor; (2) the constitutive activity of L834R deems the mutant to be a more potent target for therapeutic inhibition; (3) The differences in the predicted downstream response (between the wildtype and the mutant) accounted for by early signaling events can potentially lead to a divergent long-term behavior in the two systems causing a differential sensitivity to inhibition. More conclusive inferences from our modeling results can only be obtained after we consider the effect of other receptor states (namely, one in which Y845 is phosphorylated) and interactions with other Erb partners (see below), on down-stream signal response.

In conclusion, we have described the role of a hierarchical multiscale computational approach in resolving at a molecular level the systems-level model of EGFR-mediated signal transduction, to help rationalize the collective information emerging from biochemical, cell biology, and clinical studies. Our approach enables us to transcribe differences in molecular mechanisms as well as functional activity originating at the single molecule level into tangible differences in early signaling events. Therefore, subject to the well appreciated modeling limitations discussed in Section S1.6, this predictive capability is useful in differentiating signaling characteristics of mutant cell lines from the native wildtype case. This molecular-based refinement of the overall EGFR signal transduction model is a first attempt at untangling the differential nature of early signaling events triggered by receptor activation and their consequences in shaping the signal transduction in altered cell lines derived from different pathologies. A complete model, which is currently being pursued, will not only require resolving the differential characteristics of all of the tyrosine phosphorylation sites in EGFRTK and their associated substrate recognition properties in EGFR, but also the extension to other Erb family members in the context of homoand hetero-dimers. Transactivation of EGFR occurring through ligand-induced receptor heterodimer- 
ization $^{68}$ combined with a potential for differential signaling adds a palette of finer control elements in the ErbB-family signaling network. Indeed, recent studies have identified possible mutations in ErbB2 that may correlate with drug sensitivity in different cancers. ${ }^{40,41}$ The computational tools described here are ideal for assessing the likely effect of novel EGFR and ErbB2 mutations and determining whether the drug-sensitizing mutations implicated in nonsmall-cell lung cancer also occur in other cancers. We believe that our model driven approach will in the long-term significantly impact the optimization of future small molecule therapeutic inhibition strategies.

\section{ACKNOWLEDGMENTS}

We are indebted to Mark A Lemmon for inspiring us to work on this problem and for numerous discussions. We also thank Ms. Sung-hee Choi for her input. Funding for this work was in part from the Whitaker Foundation, and a NIH training grant through the Genomics and Computational Biology program at the University of Pennsylvania. Computational resources were provided in part by the National Center for Supercomputing Alliance under Grant DAC1103423 and National Partnership for Advanced Computational Infrastructure under Grant MCB060006.

\section{ELECTRONIC SUPPLEMENTARY MATERIAL}

The online version of this article (doi:10.1007/ s10439-006-9251-0) contains supplementary material, which is available to authorized users.

\section{REFERENCES}

\footnotetext{
${ }^{1}$ Albeck, J. G. et al. Collecting and organizing systematic sets of protein data Nat. Rev. Mol. Cell Biol. 7(11):803812, 2006.

${ }^{2}$ Arteaga, C. L. Epidermal growth factor receptor dependence in human tumors: More than just expression? Oncologist 7(Suppl. 4):31-39, 2002.

${ }^{3}$ Arteaga, C. L., and C. I. Truica. Challenges in the development of anti-epidermal growth factor receptor therapies in breast cancer Semin. Oncol. 31(1 Suppl. 3):3-8, 2004.

${ }^{4}$ Bhalla, U. S. and R. Iyengar. Emergent properties of biological networks Science 283:381-387, 1999.

${ }^{5}$ Brignola, P. S. et al. Comparison of biochemical and kinetic properties of type 1 receptor tyrosine kinase intracellular domains J. Biol. Chem. 277:1576-1581, 2001. ${ }^{6}$ Brooks, B. R. et al. Charmm - a program for macromolecular energy, minimization, and dynamics calculations J. Comput. Chem. 4(2):187-217, 1983.

${ }^{7}$ Brown, K. S. et al. The statistical mechanics of complex signaling networks: Nerve growth factor signaling Phys. Biol. 1:184-195, 2004.
}

${ }^{8}$ Burgess, A. W. et al. An open-and-shut case? Recent insights into the activation of EGF/ErbB receptors Mol. Cell 12:541-552, 2003.

${ }^{9}$ Carey, K. D. et al. Kinetic analysis of epidermal growth factor receptor somatic mutant proteins shows increased sensitivity to the epidermal growth factor receptor tyrosine kinase inhibitor, erlotinib Cancer Res. 66(16):8163-8171, 2006.

${ }^{10}$ Chen, Y. R. et al. Distinctive activation patterns in constitutively active and gefitinib-sensitive EGFR mutants Oncogene 25(8):1205-1215, 2006.

${ }^{11}$ Choi, S. H., J. M. Mendrola, and M. A. Lemmon. EGFindependent activation of cell-surface EGF receptors harboring mutations found in gefitinib-sensitive lung cancer. Oncogene advance online publication 2006.

${ }^{12} \mathrm{Chou}, \mathrm{T}$. Y. et al. Mutation in the tyrosine kinase domain of epidermal growth factor receptor is a predictive and prognostic factor for gefitinib treatment in patients with non-small cell lung cancer Clin. Cancer Res. 11(10):37503757, 2005.

${ }^{13}$ Ciardiello, F. Epidermal growth factor receptor tyrosine kinase inhibitors as anticancer agents [Review] [44 refs]. Drugs 60:25-32, 2000.

${ }^{14}$ Ciardiello, F. et al. Inhibition of growth factor production and angiogenesis in human cancer cells by ZD1839 (Iressa), a selective epidermal growth factor receptor tyrosine kinase inhibitor Clin. Cancer Res. 7(5):1459-1465, 2001.

${ }^{15}$ Ciardiello, F. et al. ZD1839 (IRESSA), an EGFRselective tyrosine kinase inhibitor, enhances taxane activity in bcl-2 overexpressing, multidrug-resistant MCF7 ADR human breast cancer cells Int. J. Cancer 98(3):463-469, 2002.

${ }^{16}$ Ciardiello, F. et al. The role of EGFR inhibitors in nonsmall cell lung cancer [Review] [54 refs]. Curr. Opin. Oncol. 16(2):130-135, 2004.

${ }^{17}$ Ciardiello, F. and G. Tortora. A novel approach in the treatment of cancer: Targeting the epidermal growth factor receptor [Review] [101 refs]. Clin. Cancer Res. 7(10):29582970, 2001.

${ }^{18}$ Citri, A. and Y. Yarden. EGF-ERBB signalling: towards the systems level Nat. Rev. Mol. Cell Biol. 7(7):505-516, 2006.

${ }^{19}$ Couzin, J. Cancer drugs - Smart weapons prove tough to design Science 298(5593):522-+, 2002.

${ }^{20}$ Dancey, J. Epidermal growth factor receptor inhibitors in clinical development [Review] [40 refs]. Int. J. Radiat. Oncol. Biol. Phys. 58(3):1003-1007, 2004.

${ }^{21}$ Dancey, J. E. Predictive factors for epidermal growth factor receptor inhibitors-the bull's-eye hits the arrow [Review] [31 refs]. Cancer Cell 5(5):411-415, 2004.

${ }^{22}$ Dill, K. A. Molecular Driving Forces. Garland Science, New York, 614 pp, 2003

${ }^{23}$ Fan, Y. X. et al. Ligand regulates epidermal growth factor receptor kinase specificity - Activation increases preference for GAB1 and SHC versus autophosphorylation sites J. Biol. Chem. 279(37):38143-38150, 2004.

${ }^{24}$ Farsad, K. et al. Generation of high curvature membranes mediated by direct endophilin bilayer interactions $J$. Cell Biol. 155:193-200, 2001.

${ }^{25}$ Ferguson, K. M. et al. EGF activates its receptor by removing interactions that autoinhibit ectodomain dimerization Mol. Cell 11:507-517, 2003.

${ }^{26}$ Ford, M. G. J. et al. Curvature of clathrin coated pits driven by Epsin Nature 419:361-366, 2002.

${ }^{27}$ Gotoh, N. et al. A highly conserved tyrosine residue at codon 845 within the kinase domain is not required for the 
transforming activity of human epidermal growth-factor receptor Biochem. Biophys. Res. Commun. 186:768-774, 1992.

${ }^{28}$ Grubmüller, H. et al. A small molecular-kianse interaction map for clinical kinase inhibitors Nat. Biotechnol. 3:329336, 2005.

${ }^{29}$ Hubbard, S. R., and J. H. Till. Tyrosine kinase structure and function Annu. Rev. Biochem. 69:373-398, 2000.

${ }^{30}$ Humphrey, W., A. Dalke, and K. Schulten. VMD - visual molecular dynamics J. Mol. Graph. 14:33-38, 1996.

${ }^{31}$ Janes, K. A. et al. A systems model of signaling identifies a molecular basis set for cytokine-induced apoptosis Science 310(5754):1646-1653, 2005.

${ }^{32}$ Janes, K. A. et al. The response of human epithelial cells to TNF involves an inducible autocrine cascade Cell 124(6):1225-1239, 2006

${ }^{33}$ Janes, K. A., and D. A. Lauffenburger. A biological approach to computational models of proteomic networks Curr. Opin. Chem. Biol. 10(1):73-80, 2006.

${ }^{34}$ Janmaat, M. L. et al. Response to epidermal growth factor receptor inhibitors in non-small cell lung cancer cells: limited antiproliferative effects and absence of apoptosis associated with persistent activity of extracellular signalregulated kinase or Akt kinase pathways Clin. Cancer Res. 9(6):2316-2326, 2003.

${ }^{35}$ Jarvis, L. M. Battling breast cancer Chem. Eng. News 84(32):21-27, 2006.

${ }^{36}$ Jorrinssen, R. N. Epidermal growth factor receptor: Mechanism of activation and signaling Exp. Cell. Res. 284:31-53, 2003.

${ }^{37}$ Kholodenko, B. N. et al. Quantification of short-term signaling by EGFR J. Biol. Chem. 274:30169-30181, 2004.

${ }^{38}$ Kobayashi, S., T. J. Boggon, T. Dayaram, P. A. Jänne, O. Kocher, M. Meyerson, B. E. Johnson, M. J. Eck, D. G. Tenen, and B. Halmos. EGFR mutation and resistance of non-small-cell lung cancer to gefitinib. N. Engl. J. Med. 352(8):786-792, 2005.

${ }^{39}$ Krauss, G. Biochemistry of Signal Transduction and Regulation. 3rd completely rev. ed. Weinheim: WileyVCH, xvi, 2003, 541 p.

${ }^{40}$ Lee, J. W. et al. ERBB2 kinase domain mutation in a gastric cancer metastasis APMIS 113(10):683-687, 2005.

${ }^{41}$ Lee, J. W. et al. Somatic mutations of ERBB2 kinase domain in gastric, colorectal, and breast carcinomas Clin. Cancer Res. 12(1):57-61, 2006.

${ }^{42}$ Lynch, T. J. et al. Activating mutations in the epidermal growth factor receptor underlying responsiveness of nonsmall-cell lung cancer to gefitinib [see comment]. N. Engl. J. Med. 350(21):2129-2139, 2004.

${ }^{43}$ Mendelsohn, J. and J. Baselga. The EGF receptor family as targets for cancer therapy Oncogene 19(56):6550-6565, 2000.

${ }^{44}$ Mildvan, A. S. Mechanisms of signaling and related enzymes Proteins Struct. Funt. Gen. 29:401-416, 1997.

${ }^{45}$ Morris, G. M. et al. Automated docking using a lamarckian genetic algorithm and and empirical binding free energy function J. Comput. Chem. 19:1639-1662, 1998.

${ }^{46}$ Moulder, S. L. et al. Epidermal growth factor receptor (HER1) tyrosine kinase inhibitor ZD1839 (Iressa) inhibits HER2/neu (erbB2)-overexpressing breast cancer cells in vitro and in vivo Cancer Res. 61(24):8887-8895, 2001.

${ }^{47}$ Nolen, B., S. Taylor, and G. Ghosh. Regulation of protein kinases: controlling activity through activation segment conformation Mol. Cell 15(5):661-675, 2004.

${ }^{48}$ Paez, J. G. et al. EGFR mutations in lung cancer: Correlation with clinical response to gefitinib therapy [see comment]. Science 304(5676):1497-1500, 2004.
${ }^{49}$ Pao, W. and V. A. Miller. Epidermal growth factor receptor mutations, small-molecule kinase inhibitors, and non small-cell lung cancer: Current knowledge and future directions. J. Clin. Oncol. 23:2556-2568, 2005.

${ }^{50}$ Petrelli, A. et al. The endophilin-CIN85-Cbl complex mediates ligand-dependent downregulation of c-Met Nature 416:187-190, 2002

${ }^{51}$ Ritter, C. A. and C. L. Arteaga. The epidermal growth factor receptor-tyrosine kinase: A promising therapeutic target in solid tumors [Review] [69 refs]. Semin. Oncol. 30(1 Suppl 1):3-11, 2003.

${ }^{52}$ Saso, K. et al. Differential inhibition of epidermal growth factor signaling pathways in rat hepatocytes by long-term ethanol treatment Gastroenterology 112(6):2073-2088, 1997.

${ }^{53}$ Schlessinger, J Cell signaling by receptor tyrosine kinases Cell 103:211-225, 2000.

${ }^{54}$ Schlessinger, J Common and distinct elements in cellular signaling between $\mathrm{EGF}$ and $\mathrm{FGF}$ receptors Science 306:1506-1507, 2004.

${ }^{55}$ Schmid, S. L. Clathrin-coated vesicle formation and protein sorting Ann. Rev. Biochem. 66:511-548, 1997.

${ }^{56} \mathrm{Schmidt}$, A. et al. Endophilin $\sim$ I mediates synaptic vesicle formation by transfer of arachidonate to lysophosphatidic acid Nature 401:133-141, 1999.

${ }^{57}$ Schoeberl, B. et al. Computational modeling of the dynamics of MAPKinase cascade activated by surface and internalized receptors Nat. Biotechnol. 20:370-375, 2002.

${ }^{58}$ Shawver, L. K., D. Slamon, and A. Ullrich. Smart drugs: Tyrosine kinase inhibitors in cancer therapy Cancer Cell 1:117-123, 2002.

${ }^{59}$ Sneyd, J. and J. F. Dufour. A dynamic model of the type-2 inositol trisphosphate receptor Proc. Natl. Acad. Sci. U.S.A. 99(4):2398-2403, 2002.

${ }^{60}$ Songyang, Z. et al. Catalytic specificity of protein-tyrosine kinases is critical for selective signalling Nature 373:536539, 1995.

${ }^{61}$ Sordella, R. et al. Gefitinib-sensitizing EGFR mutations in lung cancer activate anti-apoptotic pathways Science 305(5687):1163-1167, 2004.

${ }^{62}$ Soubeyran, P. et al. Cbl-CIN85-endophilin complex mediates ligand-induced downregulation of EGF receptors Nature 416:183-187, 2002.

${ }^{63}$ Stamos, J., M. X. Sliwkowski, and C. Eigenbrot. Structure of the epidermal growth factor receptor kinase domain alone and in complex with a 4-anilinoquinazoline inhibitor J. Biol. Chem. 277(48):46265-46272, 2002.

${ }^{64}$ Steitz, T. A. et al. A unified polymerase mechanism for nonhomologous DNA and RNA polymerases Science 266:2022-2025, 1994.

${ }^{65}$ Tracy, S. et al. Gefitinib induces apoptosis in the EGFRL858R non-small-cell lung cancer cell line H3255 Cancer Res. 64(20):7241-7244, 2004.

${ }^{66}$ Traxler, P. et al. Use of a pharmacophore model for the design of EGFR tyrosine kinase inhibitors: Isoflavones and 3-phenyl-4(1H)-quinolones $\quad J$. Med. Chem. 42(6):10181026, 1999.

${ }^{67}$ Traxler, P. and P. Furet. Strategies toward the design of novel and selective protein tyrosine kinase inhibitors Pharmacol. Ther. 82(2-3):195-206, 1999.

${ }^{68}$ Waterman, H. and Y. Yarden. Molecular mechanisms underlying endocytosis and sorting of Erb receptor tyrosine kinases FEBS lett. 490:142-152, 2001.

${ }^{69}$ Weinstein, J. and R. Radhakrishnan. A coarse-grained methodology for simulating interfacial dynamics in com- 
plex fluids: Application to protein mediated membrane processes. Mol. Phys. (2006 in press).

${ }^{70}$ Wolf-Yadlin, A. et al. Effects of HER2 overexpression on cell signaling networks governing proliferation and migration Mol. Syst. Biol. 2:54.

${ }^{71}$ Wood, E. R. et al. A unique structure for epidermal growth factor receptor bound to GW572016 (Lapatinib) Cancer Res. 64:6652-6659, 2004.

${ }^{72}$ Yarden, Y. and M. X. Sliwkowski. Untangling the ErbB signaling network Nat. Rev. Mol. Cell Biol. 2:127-137, 2001.
${ }^{73}$ Ybe, J. A. et al. Clathrin self-assembly is mediated by a tandemly repeated superhelix Nature 399:317-375, 1999.

${ }^{74}$ Zhang, L., P. Carroll, and E. Meggers. Ruthenium complexes as protein kinase inhibitors Org. Lett. 6(4):521-523, 2004.

${ }^{75}$ Zhang, X. et al. An allosteric mechanism for activation of the kinase domain of epidermal growth factor receptor Cell 125(6):1137-1149, 2006 